\title{
The Lyapunov dimension and its estimation via the Leonov method.
}

\author{
N.V. Kuznetsov \\ ${ }^{a}$ Faculty of Mathematics and Mechanics, St. Petersburg State University, Russia \\ ${ }^{b}$ Department of Mathematical Information Technology, University of Jyväskylä, Finland
}

\begin{abstract}
Along with widely used numerical methods for estimating and computing the Lyapunov dimension there is an effective analytical approach, proposed by G.A. Leonov in 1991. The Leonov method is based on the direct Lyapunov method with special Lyapunov-like functions. The advantage of the method is that it allows one to estimate the Lyapunov dimension of invariant sets without localization of the set in the phase space and, in many cases, to get effectively an exact Lyapunov dimension formula.

In this work the invariance of the Lyapunov dimension with respect to diffeomorphisms and its connection with the Leonov method are discussed. For discrete-time dynamical systems an analog of Leonov method is suggested. In a simple but rigorous way, here it is presented the connection between the Leonov method and the key related works: Kaplan and Yorke (the concept of the Lyapunov dimension, 1979), Douady and Oesterlé (upper bounds of the Hausdorff dimension via the Lyapunov dimension of maps, 1980), Constantin, Eden, Foiaş, and Temam (upper bounds of the Hausdorff dimension via the Lyapunov exponents and Lyapunov dimension of dynamical systems, 1985-90), and the numerical calculation of the Lyapunov exponents and dimension.

Keywords: attractor, Hausdorff dimension, Lyapunov dimension and its Kaplan-Yorke formula, finite-time Lyapunov exponents, invariance with respect to diffeomorphisms, Leonov method
\end{abstract}

\section{Introduction}

The concept of the Lyapunov dimension was suggested in the seminal paper by Kaplan and Yorke [36] for estimating the Hausdorff dimension of attractors. The direct numerical computation of the Hausdorff dimension of attractors is often a problem of high numerical complexity (see, e.g. discussion in [74]), thus, various estimates of this dimension are of interest. Later the concept of the Lyapunov dimension has been developed in a number of papers (see, e.g. [14, 24, 26, 28, 32, 35, 45, 78] and others).

Along with widely used numerical methods for estimating and computing the Lyapunov dimension there is an effective analytical approach, proposed by Leonov in 1991 [60] (see also [46, 50, 55, 62, 63]). The Leonov method is based on the direct Lyapunov method with special Lyapunov-like functions. The advantage of Leonov method is that it allows one to estimate the Lyapunov dimension of invariant sets without localization of the set in the phase space and in many cases to get effectively exact Lyapunov dimension formula [46, 47, 50, 53, 54, 56, 59, 64.

Further the invariance of the Lyapunov dimension with respect to diffeomorphisms and its connection with the Leonov method are discussed. For discrete-time dynamical systems an analog of Leonov method is suggested.

Email address: nkuznetsov239@gmail.com (N.V. Kuznetsov) 


\section{Lyapunov dimension of maps and dynamical systems}

Consider an autonomous differential equation

$$
\dot{u}=f(u), \quad f: U \subseteq \mathbb{R}^{n} \rightarrow \mathbb{R}^{n}
$$

where $f$ is a continuously differentiable vector-function. Suppose that any solution $u\left(t, u_{0}\right)$ of (1) such that $u\left(0, u_{0}\right)=u_{0} \in U$ exists for $t \in[0, \infty)$, it is unique and stays in $U$. Then the evolutionary operator $\varphi^{t}\left(u_{0}\right)=u\left(t, u_{0}\right)$ is continuously differentiable and satisfies the semigroup property:

$$
\varphi^{t+s}\left(u_{0}\right)=\varphi^{t}\left(\varphi^{s}\left(u_{0}\right)\right), \varphi^{0}\left(u_{0}\right)=u_{0} \quad \forall t, s \geq 0, \forall u_{0} \in U .
$$

Thus, $\left\{\varphi^{t}\right\}_{t \geq 0}$ is a smooth dynamical system in the phase space $(U,\|\cdot\|):\left(\left\{\varphi^{t}\right\}_{t \geq 0},\left(U \subseteq \mathbb{R}^{n},\|\cdot\|\right)\right)$. Here $\|u\|=\sqrt{u_{1}^{2}+\cdots+u_{n}^{2}}$ is Euclidean norm of the vector $u=\left(u_{1}, \ldots, u_{n}\right) \in \mathbb{R}^{n}$. Similarly, one can consider a dynamical system generated by the difference equation

$$
u(t+1)=\varphi(u(t)), \quad t=0,1, . .
$$

where $\varphi: U \subseteq \mathbb{R}^{n} \rightarrow U$ is a continuously differentiable vector-function. Here $\varphi^{t}(u)=\underbrace{(\varphi \circ \varphi \circ \cdots \varphi)(u)}_{t \text { times }}$, $\varphi^{0}(u)=u$, and the existence and uniqueness (in the forward-time direction) take place for all $t \geq 0$. Further $\left\{\varphi^{t}\right\}_{t \geq 0}$ denotes a smooth dynamical system with continuous or discrete time.

Consider the linearizations of systems (11) and (3) along the solution $\varphi^{t}(u)$ :

$$
\begin{gathered}
\dot{y}=J\left(\varphi^{t}(u)\right) y, \quad J(u)=D f(u), \\
y(t+1)=J\left(\varphi^{t}(u)\right) y(t), \quad J(u)=D \varphi(u),
\end{gathered}
$$

where $J(u)$ is the $n \times n$ Jacobian matrix, the elements of which are continuous functions of $u$. Suppose that $\operatorname{det} J(u) \neq 0 \forall u \in U$.

Consider the fundamental matrix, which consists of linearly independent solutions $\left\{y^{i}(t)\right\}_{i=1}^{n}$ of the linearized system,

$$
D \varphi^{t}(u)=\left(y^{1}(t), \ldots, y^{n}(t)\right), \quad D \varphi^{0}(u)=I
$$

where $I$ is the unit $n \times n$ matrix. An important cocycle property of fundamental matrix (6) is as follows

$$
D \varphi^{t+s}(u)=D \varphi^{t}\left(\varphi^{s}(u)\right) D \varphi^{s}(u), \forall t, s \geq 0, \forall u \in U .
$$

Let $\sigma_{i}(t, u)=\sigma_{i}\left(D \varphi^{t}(u)\right), i=1,2, . ., n$, be the singular values of $D \varphi^{t}(u)$ (i.e. $\sigma_{i}(t, u)>0$ and $\sigma_{i}(t, u)^{2}$ are the eigenvalues of the symmetric matrix $D \varphi^{t}(u)^{*} D \varphi^{t}(u)$ with respect to their algebraic multiplicity), ordered so that $\sigma_{1}(t, u) \geq \cdots \geq \sigma_{n}(t, u)>0$ for any $u \in U, t \geq 0$. The singular value function of order $d \in[0, n]$ at the point $u \in U$ for $D \varphi^{t}(u)$ is defined as

$$
\omega_{d}\left(D \varphi^{t}(u)\right)= \begin{cases}1, & d=0, \\ \sigma_{1}(t, u) \sigma_{2}(t, u) \cdots \sigma_{d}(t, u), & d \in\{1,2, . ., n\}, \\ \sigma_{1}(t, u) \cdots \sigma_{\lfloor d\rfloor}(t, u) \sigma_{\lfloor d\rfloor+1}(u)^{d-\lfloor d\rfloor}, & d \in(0, n),\end{cases}
$$

where $\lfloor d\rfloor$ is the largest integer less or equal to $d$. Remark that $\left|\operatorname{det} D \varphi^{t}(u)\right|=\omega_{n}\left(D \varphi^{t}(u)\right)$. Similarly, we can introduce the singular value function for arbitrary quadratic matrices and then by the Horn inequality [31] for any two $n \times n$ matrices $A$ and $B$ and any $d \in[0, n]$ we have (see, e.g. [10, p.28])

$$
\omega_{d}(A B) \leq \omega_{d}(A) \omega_{d}(B), \quad d \in[0, n] .
$$


Let a nonempty set $K \subset U \subseteq \mathbb{R}^{n}$ be invariant with respect to the dynamical system $\left\{\varphi^{t}\right\}_{t \geq 0}$, i.e. $\varphi^{t}(K)=K$ for all $t>0$. Since in the numerical experiments only finite time $t$ can be considered, for a fixed $t \geq 0$ let us consider the map defined by the evolutionary operator $\varphi^{t}(u)$ : $\varphi^{t}: U \subseteq \mathbb{R}^{n} \rightarrow U$.

The concept of the Lyapunov dimension was suggested in the seminal paper by Kaplan and Yorke [36] and later it was developed in a number of papers (see, e.g. [14, 23, 26, 32, 45]). The following definition is inspirited by Douady and Oesterlé [20].

Definition 1. The local Lyapunov dimension 1 of the map $\varphi^{t}$ (or finite-time local Lyapunov dimension of the dynamical system $\left.\left\{\varphi^{t}\right\}_{t \geq 0}\right)$ at the point $u \in U$ is defined as

$$
\operatorname{dim}_{\mathrm{L}}\left(\varphi^{t}, u\right)=\inf \left\{d \in[0, n]: \omega_{d}\left(D \varphi^{t}(u)\right)<1\right\} .
$$

If the infimum is taken over an empty set (i.e. $\left.\omega_{n}\left(D \varphi^{t}(u)\right) \geq 1\right)$, we assume that the infimum and considered dimension are taken equal 2 to $n$.

The Lyapunov dimension of the map $\varphi^{t}$ (or finite-time Lyapunov dimension of the dynamical system $\left.\left\{\varphi^{t}\right\}_{t \geq 0}\right)$ with respect to the invariant set $K$ is defined as

$$
\operatorname{dim}_{\mathrm{L}}\left(\varphi^{t}, K\right)=\sup _{u \in K} \operatorname{dim}_{\mathrm{L}}\left(\varphi^{t}, u\right)=\sup _{u \in K} \inf \left\{d \in[0, n]: \omega_{d}\left(D \varphi^{t}(u)\right)<1\right\} .
$$

The continuity of the functions $u \mapsto \sigma_{i}\left(D \varphi^{t}(u)\right), i=1,2, ., n$, on $U$ implies that for any $d \in[0, n]$ and $t \geq 0$ the function $u \mapsto \omega_{d}\left(D \varphi^{t}(u)\right)$ is continuous on $U$ (see, e.g. [20], [27, p.554]). Therefore for a compact set $K \subset U$ and $t \geq 0$ we have

$$
\sup _{u \in K} \omega_{d}\left(D \varphi^{t}(u)\right)=\max _{u \in K} \omega_{d}\left(D \varphi^{t}(u)\right)
$$

By relation (12) for a compact invariant set $K$ one can prove that

$$
\operatorname{dim}_{\mathrm{L}}\left(\varphi^{t}, K\right)=\inf \left\{d \in[0, n]: \max _{u \in K} \omega_{d}\left(D \varphi^{t}(u)\right)<1\right\} .
$$

In the seminal paper [20] Douady and Oesterlé proved rigorously that the Lyapunov dimension of the map $\varphi^{t}$ with respect to the compact invariant set $K$ is an upper estimate of the Hausdorff dimension of the set $K$ :

$$
\operatorname{dim}_{\mathrm{H}} K \leq \operatorname{dim}_{\mathrm{L}}\left(\varphi^{t}, K\right) .
$$

For numerical estimations of dimension, the following remark is important. From (17) and (9) it follows that

$$
\sup _{u \in K} \omega_{d}\left(D \varphi^{t+s}(u)\right)=\sup _{u \in K} \omega_{d}\left(D \varphi^{t}\left(\varphi^{s}(u)\right) D \varphi^{s}(u)\right) \leq \sup _{u \in K} \omega_{d}\left(D \varphi^{t}(u)\right) \sup _{u \in K} \omega_{d}\left(D \varphi^{s}(u)\right) \quad \forall t, s \geq 0
$$

and $\sup _{u \in K} \omega_{d}\left(D \varphi^{n t}(u)\right) \leq\left(\sup _{u \in K} \omega_{d}\left(D \varphi^{t}(u)\right)\right)^{n}$ for any integer $n \geq 0$. Thus for any $t \geq 0$ there exists $\tau=\tau(t)>0$ such that

$$
\operatorname{dim}_{\mathrm{L}}\left(\varphi^{t+\tau}, K\right) \leq \operatorname{dim}_{\mathrm{L}}\left(\varphi^{t}, K\right) .
$$

While in the computations we can consider only finite time $t$ and the map $\varphi^{t}$, from a theoretical point of view, it is interesting to study the limit behavior of finite-time Lyapunov dimension of the dynamical system $\left\{\varphi^{t}\right\}_{t \geq 0}$ with respect to the compact invariant set $K$.

\footnotetext{
${ }^{1}$ This is not a dimension in a rigorous sense (see, e.g. [4, 33, 40]). The notion 'local Lyapunov dimension' is used, e.g. in [22, 32].

${ }^{2}$ In general, since $\omega_{0}\left(D \varphi^{t}(u)\right) \equiv 1$ and $d \mapsto \omega_{d}\left(D \varphi^{t}(u)\right)$ is a left-continuous function, we have $\operatorname{dim}_{\mathrm{L}}\left(\varphi^{t}, u\right)=$ $\max \left\{d \in[0, n]: \omega_{d}\left(D \varphi^{t}(u)\right) \geq 1\right\}$. If all $\left\{\sigma_{i}(t, u)\right\}_{1}^{n}$ are assumed to be positive and $\omega_{n}\left(D \varphi^{t}(u)\right)<1$, then in (10) the infimum is achieved (see (21) and the Kaplan-Yorke formula (23)).
} 
Definition 2. The Lyapunov dimension of the dynamical system $\left\{\varphi^{t}\right\}_{t>0}$ with respect to the invariant set $K$ is defined as

$$
\operatorname{dim}_{\mathrm{L}}\left(\left\{\varphi^{t}\right\}_{t \geq 0}, K\right)=\inf _{t>0} \operatorname{dim}_{\mathrm{L}}\left(\varphi^{t}, K\right)
$$

From (14) and (13) we have

$$
\operatorname{dim}_{\mathrm{H}} K \leq \operatorname{dim}_{\mathrm{L}}\left(\left\{\varphi^{t}\right\}_{t \geq 0}, K\right)=\inf _{t>0} \sup _{u \in K} \operatorname{dim}_{\mathrm{L}}\left(\varphi^{t}, u\right)
$$

and (15) implies

$$
\inf _{t>0} \operatorname{dim}_{\mathrm{L}}\left(\varphi^{t}, K\right)=\liminf _{t \rightarrow+\infty} \operatorname{dim}_{\mathrm{L}}\left(\varphi^{t}, K\right) .
$$

Remark that if $\sup _{u \in K} \omega_{\bar{d}}\left(D \varphi^{t}(u)\right)<1$ for a certain $\bar{d} \in[0, n]$, then

$$
\inf _{t>0} \sup _{u \in K} \omega_{\bar{d}}\left(D \varphi^{t}(u)\right)=\liminf _{t \rightarrow+\infty} \sup _{u \in K} \omega_{\bar{d}}\left(D \varphi^{t}(u)\right)=0
$$

and

$$
\operatorname{dim}_{\mathrm{L}}\left(\left\{\varphi^{t}\right\}_{t \geq 0}, K\right) \leq \operatorname{dim}_{\mathrm{L}}\left(\varphi^{t}, K\right)<\bar{d}
$$

Definition 3. (see, e.g. [1]) The finite-time Lyapunov exponents (or the Lyapunov exponent functions of singular values) of the dynamical system $\left\{\varphi^{t}\right\}_{t \geq 0}$ at the point $u \in U$ are denoted by $\mathrm{LE}_{i}(t, u)=\mathrm{LE}_{i}\left(D \varphi^{t}(u)\right), i=1,2, . ., n$, and defined as

$$
\mathrm{LE}_{i}(t, u)=\frac{1}{t} \ln \sigma_{i}(t, u), \quad t>0 .
$$

Here $\mathrm{LE}_{1}(t, u) \geq \cdots \geq \mathrm{LE}_{n}(t, u)$ for all $t>0$ since the singular values are ordered by decreasing.

For the sake of simplicity, we assume that $\omega_{1}\left(D \varphi^{t}(u)\right)>1>\omega_{n}\left(D \varphi^{t}(u)\right)$ for $t>0, u \in K$. Thus, $n>\operatorname{dim}_{\mathrm{L}}\left(\varphi^{t}, u\right)>1$ and $\omega_{\operatorname{dim}_{\mathrm{L}}\left(\varphi^{t}, u\right)}\left(D \varphi^{t}(u)\right)=1$. Therefore for $j(t, u)=\left\lfloor\operatorname{dim}_{\mathrm{L}}\left(\varphi^{t}, u\right)\right\rfloor$ and $s(t, u)=\operatorname{dim}_{\mathrm{L}}\left(\varphi^{t}, u\right)-\left\lfloor\operatorname{dim}_{\mathrm{L}}\left(\varphi^{t}, u\right)\right\rfloor$ we have

$$
0=\frac{1}{t} \ln \left(\omega_{j(t, u)+s(t, u)}\left(D \varphi^{t}(u)\right)\right)=\sum_{i=1}^{j(t, u)} \operatorname{LE}_{i}(t, u)+s(t, u) \operatorname{LE}_{j(t, u)+1}(t, u) .
$$

Since $\operatorname{LE}_{i}(t, u)$ are ordered by decreasing and $s(t, u)<1$, we have

$$
\begin{aligned}
& j(t, u)=\max \left\{m: \sum_{i=1}^{m} \operatorname{LE}_{i}(t, u) \geq 0\right\}, \quad \sum_{i=1}^{j(t, u)+1} \operatorname{LE}_{i}(t, u)<0, \quad \operatorname{LE}_{j(t, u)+1}(t, u)<0, \\
& 0 \leq s(t, u)=\frac{\operatorname{LE}_{1}(t, u)+\cdots+\mathrm{LE}_{j(t, u)}(t, u)}{\left|\mathrm{LE}_{j(t, u)+1}(t, u)\right|}<1 .
\end{aligned}
$$

If $j(t, u)=0$ or $j(t, u)=n$, then let $s(t, u)=0$. The expression

$$
\operatorname{dim}_{\mathrm{L}}^{\mathrm{KY}}\left(\left\{\mathrm{LE}_{i}(t, u)\right\}_{i=1}^{n}\right)=j(t, u)+\frac{\mathrm{LE}_{1}(t, u)+\cdots+\mathrm{LE}_{j(t, u)}(t, u)}{\left|\mathrm{LE}_{j(t, u)+1}(t, u)\right|}
$$


corresponds to the Kaplan-Yorke formula [36] with respect to finite-time Lyapunov exponents (the set $\left\{\operatorname{LE}_{i}(t, u)\right\}_{1}^{n}$, ordered by decreasing 3 ). Remark that there exists $\bar{s}$ such that $s(t, u)<\bar{s}<1$ and $\omega_{j(t, u)+\bar{s}}\left(D \varphi^{t}(u)\right)<1$ for any such $\bar{s}$, and $\omega_{\underline{d}}\left(D \varphi^{t}(u)\right) \geq 1$ for $0 \leq \underline{d} \leq j(t, u)+s(t, u)$. Thus $j(t, u)+s(t, u)=\operatorname{dim}_{\mathrm{L}}\left(\varphi^{t}, u\right)$ for $j(t, u)$ and $s(t, u)$ defined by (22). Therefore, we get

Proposition 1. For the Lyapunov dimension of the map $\varphi^{t}$ (or finite-time Lyapunov dimension of the dynamical system $\left\{\varphi^{t}\right\}_{t \geq 0}$ ) with respect to the compact invariant set $K$ we have

$\operatorname{dim}_{\mathrm{H}} K \leq \sup _{u \in K} \operatorname{dim}_{\mathrm{L}}\left(\varphi^{t}, u\right)=\sup _{u \in K} \operatorname{dim}_{\mathrm{L}}^{\mathrm{KY}}\left(\left\{\operatorname{LE}_{i}(t, u)\right\}_{1}^{n}\right)=\sup _{u \in K}\left(j(t, u)+\frac{\mathrm{LE}_{1}(t, u)+\cdots+\mathrm{LE}_{j(t, u)}(t, u)}{\left|\mathrm{LE}_{j(t, u)+1}(t, u)\right|}\right)$.

For numerical computation of the finite-time Lyapunov exponent:4 there are developed various continuous and discrete algorithms based on QR and SVD decompositions of fundamental matrix (see, e.g. MATLAB realizations in [44, 55]). However such algorithms may not work well in the case of coincidence or closeness of two or more Lyapunov exponents. Also it is important to remark that numerical computation of the Lyapunov exponents can be done only for a finite time $T$, the justification of the choice of which is usually omitted, while it is known that in such computations unexpected "jumps" can occur (see, e.g. [15, p.116, Fig.6.3]). The various methods (see, e.g. [2, 30, 73, 80]) are also developed for the estimation of the Lyapunov exponents from time series. However there are known examples in which the results of such computations differ substantially from the analytical results [5, 79].

3 Various characteristics of chaotic behavior are based on the limit values of finite-time Lyapunov exponents (LEs): $\operatorname{LE}_{i}(u)=\limsup _{t \rightarrow+\infty} \mathrm{LE}_{i}(t, u), i=1, \ldots, n$. For example, Kaplan-Yorke formula with respect to LEs is considered in [14, 24 and the sum of positive LEs may be used [66, 71] as the characteristic of Kolmogorov-Sinai entropy rate (see [3, 18, 37, 77] ). Relying on ergodicity, the LEs and Lyapunov dimension of attractor are often computed along one trajectory (see also 25, 26, 36, 45]), which is attracted or belongs to the attractor. But, in general, one has to consider a grid of points on $K$ and the corresponding local Lyapunov dimensions (see, e.g. [44, 55]). For a given invariant set $K$ and a given point $u_{0} \in K$ there are two essential questions related to the computation of the Lyapunov exponents and the use of the Kaplan-Yorke formulas of local Lyapunov dimension $\sup _{u \in K} \operatorname{dim}_{\mathrm{L}}^{\mathrm{KY}}\left(\left\{\lim _{\sup _{t \rightarrow+\infty}} \mathrm{LE}_{i}(t, u)\right\}_{1}^{n}\right)$ : whether $\limsup _{t \rightarrow+\infty} \operatorname{LE}_{i}\left(t, u_{0}\right)=\lim _{t \rightarrow+\infty} \operatorname{LE}_{i}\left(t, u_{0}\right)$ is valid, and if not, whether the relation $\sup _{u \in K} \operatorname{dim}_{\mathrm{L}}^{\mathrm{KY}}\left(\left\{\mathrm{LE}_{m}(u)\right\}_{1}^{n}\right)=$ $\sup _{u \in K \backslash\left\{\varphi^{t}\left(u_{0}\right), t \geq 0\right\}} \operatorname{dim}_{\mathrm{L}}^{\mathrm{KY}}\left(\left\{\operatorname{LE}_{i}(u)\right\}_{1}^{n}\right)$ is true. In order to get rigorously the positive answer to these questions, from a theoretical point of view, one may use various ergodic properties of the dynamical system $\left\{\varphi^{t}\right\}_{t \geq 0}($ see, e.g. Oseledets [68], Ledrappier [45], and auxiliary results in [8, 17]). However, from a practical point of view, the rigorous use of the above results is a challenging task (e.g. even for the well-studied Lorenz system) and hardly can be done effectively in the general case (see, e.g. the corresponding discussions in [7], [15, p.118], 70], 81, p.9] and the works [43, 48] on the Perron effects of the largest Lyapunov exponent sign reversals). For an example of the effective rigorous use of the ergodic theory for the estimation of the Hausdorff and Lyapunov dimensions, see, e.g. [75].

4 Another widely used definition of the Lyapunov exponents goes back to Lyapunov [65]. Finite-time Lyapunov exponents $\left\{\operatorname{LCE}_{i}(t, u)\right\}_{1}^{n}$ of the fundamental matrix columns $\left(y^{1}(t, u), \ldots, y^{n}(t, u)\right)=D \varphi^{t}(u)($ called also finitetime Lyapunov characteristic exponents, LCE) are defined as the set $\left\{\frac{1}{t} \ln \left\|y^{i}(t, u)\right\|\right\}_{1}^{n}$ ordered by decreasing for $t>0$. In contrast to the definition of the Lyapunov exponents of singular values, finite-time Lyapunov exponents of fundamental matrix columns may be different for different fundamental matrices (see, e.g. [42]). To get the set of all possible values of the Lyapunov exponents of fundamental matrix columns (the set with the minimal sum of values), one has to consider the so-called normal fundamental matrices [65]. Using, e.g, Courant-Fischer theorem [31], it is possible to show that $\operatorname{LCE}_{1}(t, u)=\mathrm{LE}_{1}(t, u)$ and $\operatorname{LE}_{i}(t, u) \leq \mathrm{LCE}_{i}(t, u)$ for $1<i \leq n$, and, thus, $\operatorname{dim}_{\mathrm{L}}^{\mathrm{KY}}\left(\left\{\mathrm{LE}_{i}(t, u)\right\}_{1}^{n}\right) \leq \operatorname{dim}_{\mathrm{L}}^{\mathrm{KY}}\left(\left\{\mathrm{LCE}_{i}(t, u)\right\}_{1}^{n}\right)$. For example, for the matrix [42] $X(t)=\left(\begin{array}{cc}1 & g(t)-g^{-1}(t) \\ 0 & 1\end{array}\right)$ we have the following ordered values: $\operatorname{LCE}_{1}(X(t))=\max \left(\limsup _{t \rightarrow+\infty} \frac{1}{t} \ln |g(t)|, \limsup _{t \rightarrow+\infty} \frac{1}{t} \ln \left|g^{-1}(t)\right|\right), \mathrm{LCE} 2(X(t))=0$; $\mathrm{LE}_{1,2}(X(t))=\max , \min \left(\limsup _{t \rightarrow+\infty} \frac{1}{t} \ln |g(t)|, \limsup _{t \rightarrow+\infty} \frac{1}{t} \ln \left|g^{-1}(t)\right|\right)$.

The various generalizations of the Lyapunov exponents and their properties are studied, e.g., in [6, 11, 16, 34, 39, 48, 71]. 


\section{Invariance with respect to diffeomorphisms and analytical estimates}

While the topological dimensions are invariant with respect to Lipschitz homeomorphisms, the Hausdorff dimension is invariant with respect to Lipschitz diffeomorphisms and the noninteger Hausdorff dimension is not invariant with respect to homeomorphisms [33]. Since the Lyapunov dimension is used as an upper estimate of the Hausdorff dimension, the question arises whether the Lyapunov dimension is invariant under diffeomorphisms (see, e.g. [69]).

Consider the dynamical system $\left(\left\{\varphi^{t}\right\}_{t \geq 0},\left(U \subseteq \mathbb{R}^{n},\|\cdot\|\right)\right)$ under the change of coordinates $w=$ $h(u)$, where $h: U \subseteq \mathbb{R}^{n} \rightarrow \mathbb{R}^{n}$ is a diffeomorphism. In this case the semi-orbit $\gamma^{+}(u)=\left\{\varphi^{t}(u), t \geq\right.$ $0\}$ is mapped to the semi-orbit defined by $\varphi_{h}^{t}(w)=\varphi_{h}^{t}(h(u))=h\left(\varphi^{t}(u)\right)$, the dynamical system $\left(\left\{\varphi^{t}\right\}_{t \geq 0},\left(U \subseteq \mathbb{R}^{n},\|\cdot\|\right)\right)$ is transformed to the dynamical system $\left(\left\{\varphi_{h}^{t}\right\}_{t \geq 0},\left(h(U) \subseteq \mathbb{R}^{n},\|\cdot\|\right)\right)$, and the compact set $K \subset U$ invariant with respect to $\left\{\varphi^{t}\right\}_{t \geq 0}$ is mapped to the compact set $h(K) \subset h(U)$ invariant with respect to $\left\{\varphi_{h}^{t}\right\}_{t \geq 0}$. Here

$$
\begin{aligned}
& D_{w} \varphi_{h}^{t}(w)=D_{w}\left(h\left(\varphi^{t}\left(h^{-1}(w)\right)\right)\right)=D_{u} h\left(\varphi^{t}\left(h^{-1}(w)\right)\right) D_{u} \varphi^{t}\left(h^{-1}(w)\right) D_{w} h^{-1}(w), \\
& D_{u}\left(\varphi_{h}^{t}(h(u))\right)=D_{w} \varphi_{h}^{t}(h(u)) D_{u} h(u)=D_{u}\left(h\left(\varphi^{t}(u)\right)\right)=D_{u} h\left(\varphi^{t}(u)\right) D_{u} \varphi^{t}(u) .
\end{aligned}
$$

Therefore

$$
D_{w} h^{-1}(w)=\left(D_{u} h(u)\right)^{-1}
$$

and

$$
D \varphi_{h}^{t}(w)=D h\left(\varphi^{t}(u)\right) D \varphi^{t}(u)(D h(u))^{-1}
$$

If $u \in K$, then $\varphi^{t}(u)$ and $\varphi_{h}^{t}(h(u))$ define bounded semi-orbits. Remark that $D h$ and $(D h)^{-1}$ are continuous and, thus, $D h\left(\varphi^{t}(u)\right)$ and $\left(D h\left(\varphi^{t}(u)\right)\right)^{-1}$ are bounded in $t$. From (12) it follows that for any $d \in[0, n]$ there is a constant $c=c(d) \geq 1$ such that (see also [10, p.29])

$$
\max _{u \in K} \omega_{d}(D h(u)) \leq c, \quad \max _{u \in K} \omega_{d}\left((D h(u))^{-1}\right) \leq c .
$$

Lemma 1. If for $t>0$ there exist diffeomorphism $h: U \subseteq \mathbb{R}^{n} \rightarrow \mathbb{R}^{n}$ and $d \in[0, n]$ such that the estimation 5

$$
\max _{w \in h(K)} \omega_{d}\left(D \varphi_{h}^{t}(w)\right)=\max _{u \in K} \omega_{d}\left(D h\left(\varphi^{t}(u)\right) D \varphi^{t}(u)(D h(u))^{-1}\right)<1
$$

is valid, then for $u \in K$ we get

$$
\liminf _{t \rightarrow+\infty}\left(\omega_{d}\left(D \varphi^{t}(u)\right)-\omega_{d}\left(D \varphi_{h}^{t}(h(u))\right)\right)=0
$$

and

$$
\liminf _{t \rightarrow+\infty} \omega_{d}\left(D \varphi_{h}^{t}(h(u))\right)=\liminf _{t \rightarrow+\infty} \omega_{d}\left(D \varphi^{t}(u)\right)=0 .
$$

Proof. Applying (9) to (24), we get

$$
\omega_{d}\left(D \varphi_{h}^{t}(h(u))\right) \leq \omega_{d}\left(D h\left(\varphi^{t}(u)\right)\right) \omega_{d}\left(D \varphi^{t}(u)\right) \omega_{d}\left((D h(u))^{-1}\right) .
$$

By (25) we obtain

$$
\omega_{d}\left(D \varphi_{h}^{t}(h(u))\right) \leq c^{2} \omega_{d}\left(D \varphi^{t}(u)\right)
$$

\footnotetext{
${ }^{5}$ The expression in (26) corresponds to the expressions considered in [60, eq.(1)] for $p(u)=D h(u)$, [46, eq. $\left.(1)\right]$ and [61, p.99, eq.10.1] for $Q(u)=D h(u)$.
} 
Similarly,

$$
\omega_{d}\left(D \varphi^{t}(u)\right) \leq \omega_{d}\left(\left(D h\left(\varphi^{t}(u)\right)\right)^{-1}\right) \omega_{d}\left(D \varphi_{h}^{t}(h(u))\right) \omega_{d}(D h(u))
$$

and

$$
\omega_{d}\left(D \varphi^{t}(u)\right) \leq c^{2} \omega_{d}\left(D \varphi_{h}^{t}(h(u))\right) .
$$

Therefore for any $d \in[0, n], t \geq 0$, and $u \in K$ we have

$$
c^{-2} \omega_{d}\left(D \varphi_{h}^{t}(h(u))\right) \leq \omega_{d}\left(D \varphi^{t}(u)\right) \leq c^{2} \omega_{d}\left(D \varphi_{h}^{t}(h(u))\right)
$$

and

$$
\left(c^{-2}-1\right) \omega_{d}\left(D \varphi_{h}^{t}(h(u))\right) \leq \omega_{d}\left(D \varphi^{t}(u)\right)-\omega_{d}\left(D \varphi_{h}^{t}(h(u))\right) \leq\left(c^{2}-1\right) \omega_{d}\left(D \varphi_{h}^{t}(h(u))\right) .
$$

If for $t \geq 0$ there exists $d \in[0, n]$ such that $\sup _{u \in K} \omega_{d}\left(D \varphi_{h}^{t}(h(u))\right)<1$ (see (26)), then by (19)) we get

$$
\liminf _{t \rightarrow+\infty} \omega_{d}\left(D \varphi_{h}^{t}(h(u))\right)=0
$$

and

$$
0 \leq \liminf _{t \rightarrow+\infty}\left(\omega_{d}\left(D \varphi^{t}(u)\right)-\omega_{d}\left(D \varphi_{h}^{t}(h(u))\right)\right) \leq 0 .
$$

Corollary 1. (see, e.g. [42]) For $u \in K$ we have

$$
\lim _{t \rightarrow+\infty}\left(\operatorname{LE}_{i}\left(D \varphi_{h}^{t}(h(u))\right)-\operatorname{LE}_{i}\left(D \varphi^{t}(u)\right)\right)=0, \quad i=1,2, . ., n,
$$

and, therefore,

$$
\limsup _{t \rightarrow+\infty} \operatorname{LE}_{i}\left(D \varphi_{h}^{t}(h(u))\right)=\limsup _{t \rightarrow+\infty} \operatorname{LE}_{i}\left(D \varphi^{t}(u)\right), \quad i=1,2, . ., n .
$$

Proof. From (27) for $t>0$ we obtain

$$
\frac{1}{t} \ln c^{-2}+\frac{1}{t} \ln \omega_{d}\left(D \varphi_{h}^{t}(h(u))\right) \leq \frac{1}{t} \ln \omega_{d}\left(D \varphi^{t}(u)\right) \leq \frac{1}{t} \ln c^{2}+\frac{1}{t} \ln \omega_{d}\left(D \varphi_{h}^{t}(h(u))\right) .
$$

Thus for the integer $d=m$ we have

$$
\lim _{t \rightarrow+\infty}\left(\frac{1}{t} \ln \omega_{m}\left(D \varphi^{t}(u)\right)-\frac{1}{t} \ln \omega_{m}\left(D \varphi_{h}^{t}(h(u))\right)\right)=\lim _{t \rightarrow+\infty}\left(\sum_{i=1}^{m} \operatorname{LE}_{i}\left(D \varphi^{t}(u)\right)-\sum_{i=1}^{m} \operatorname{LE}_{i}\left(D \varphi_{h}^{t}(h(u))\right)\right)=0
$$

The above statements are reformulations from [42, 47] and imply the following

Proposition 2. The Lyapunov dimension of the dynamical system $\left\{\varphi^{t}\right\}_{t \geq 0}$ with respect to the compact invariant set $K$ is invariant with respect to any diffeomorphism $h: U \subseteq \mathbb{R}^{n} \rightarrow \mathbb{R}^{n}$, i.e.

$$
\operatorname{dim}_{\mathrm{L}}\left(\left\{\varphi^{t}\right\}_{t \geq 0}, K\right)=\operatorname{dim}_{\mathrm{L}}\left(\left\{\varphi_{h}^{t}\right\}_{t \geq 0}, h(K)\right) .
$$

Proof. Lemma 1 implies that if $\max _{w \in h(K)} \omega_{d}\left(D \varphi_{h}^{t}(w)\right)<1$ for $t>0$ and $d \in[0, n]$, then there exists $T>t$ such that

$$
\max _{u \in K} \omega_{d}\left(D \varphi^{T}(u)\right)<1
$$

and vice verse. Thus, from (201), we have $\operatorname{dim}_{\mathrm{L}}\left(\left\{\varphi^{t}\right\}_{t \geq 0}, K\right)<d \Leftrightarrow \operatorname{dim}_{\mathrm{L}}\left(\left\{\varphi_{h}^{t}\right\}_{t \geq 0}, h(K)\right)<d$.

Remark that the invariance with respect to Lipschitz diffeomorphisms is an essential point for the introduction of the Lyapunov dimension on manifolds. 
Corollary 2. Suppose $H(u)$ is an $n \times n$ matrix, all elements of which are scalar continuous functions of $u$, and $\operatorname{det} H(u) \neq 0$ for all $u \in K$. If for $t>0$ there exists $d \in(0, n]$ such that

$$
\max _{u \in K} \omega_{d}\left(H\left(\varphi^{t}(u)\right) D \varphi^{t}(u)(H(u))^{-1}\right)<1,
$$

then by (26) with $D h(u)=H(u)$, (29), and (30), for all sufficiently large $T>0$ we have

$$
\operatorname{dim}_{\mathrm{H}} K \leq \operatorname{dim}_{\mathrm{L}}\left(\left\{\varphi^{t}\right\}_{t \geq 0}, K\right) \leq \operatorname{dim}_{\mathrm{L}}\left(\varphi^{T}, K\right)<d .
$$

If we take $H(u)=p(u) S$, where $p: U \subseteq \mathbb{R}^{n} \rightarrow \mathbb{R}^{1}$ is a continuous positive scalar function, $p(u) \neq 0$ for all $u \in K \subset U$, and $S$ is a nonsingular $n \times n$ matrix, condition (31) takes the form

$$
\sup _{u \in K} \omega_{d}\left(H\left(\varphi^{t}(u)\right) D \varphi^{t}(u)(H(u))^{-1}\right)=\sup _{u \in K}\left(\left(p\left(\varphi^{t}(u)\right) p(u)^{-1}\right)^{d} \omega_{d}\left(S D \varphi^{t}(u) S^{-1}\right)\right)<1 .
$$

Remark that if a nonsingular matrix $S$ is such that

$$
S D \varphi^{t}(u) S^{-1}=\operatorname{diag}\left(\lambda_{1}(t, u), . ., \lambda_{n}(t, u)\right), \quad\left|\lambda_{1}(t, u)\right| \geq . . \geq\left|\lambda_{n}(t, u)\right|,
$$

then $\sigma_{i}\left(S D \varphi^{t}(u) S^{-1}\right)=\left|\lambda_{i}(t, u)\right|$.

Let us apply the linear change of variables $w=h(u)=S u$ with a nonsingular $n \times n$ matrix $S$. Then $\varphi^{t}\left(u_{0}\right)=u\left(t, u_{0}\right)$ is transformed into $\varphi_{S}^{t}\left(w_{0}\right)$

$$
\varphi_{S}^{t}\left(w_{0}\right)=w\left(t, w_{0}\right)=S \varphi^{t}\left(u_{0}\right)=S u\left(t, S^{-1} w_{0}\right) .
$$

Consider transformed systems (11) and (3)

$$
\dot{w}=S f\left(S^{-1} w\right) \text { or } w(t+1)=S \varphi\left(S^{-1} w(t)\right)
$$

and their linearizations along the solution $\varphi_{S}^{t}\left(w_{0}\right)=w\left(t, w_{0}\right)=S \varphi^{t}\left(u_{0}\right)$ :

$$
\begin{aligned}
& \dot{v}=J_{S}\left(w\left(t, w_{0}\right)\right) v \quad \text { or } \quad v(t+1)=J_{S}\left(w\left(t, w_{0}\right)\right) v(t), \\
& J_{S}\left(w\left(t, w_{0}\right)\right)=S J\left(S^{-1} w\left(t, w_{0}\right)\right) S^{-1}=S J\left(u\left(t, u_{0}\right)\right) S^{-1} .
\end{aligned}
$$

For the corresponding fundamental matrices we have $D \varphi_{S}^{t}(w)=S D \varphi^{t}(u) S^{-1}$.

Proposition 3. Suppose that at one of the equilibrium points of the dynamical system $\left\{\varphi^{t}\right\}_{t \geq 0}$ : $u_{e q} \equiv \varphi^{t}\left(u_{e q}\right), u_{e q} \in U$, the matrix $J\left(u_{e q}\right)$ has simple real eigenvalues: $\left\{\lambda_{i}\left(u_{e q}\right)\right\}_{i=1}^{n}$. Consider a nonsingular matrix $S$ such that

$$
S J\left(u_{e q}\right) S^{-1}=\operatorname{diag}\left(\lambda_{1}\left(u_{e q}\right), . ., \lambda_{n}\left(u_{e q}\right)\right),
$$

where $\lambda_{i}\left(u_{e q}\right) \geq \lambda_{i+1}\left(u_{e q}\right)$ for the case of continuous-time dynamical systems and $\left|\lambda_{i}\left(u_{e q}\right)\right| \geq$ $\left|\lambda_{i+1}\left(u_{e q}\right)\right|$ for discrete-time dynamical systems. Then

$$
\operatorname{dim}_{\mathrm{L}}\left(\left\{\varphi^{t}\right\}_{t \geq 0}, u_{e q}\right)=\operatorname{dim}_{\mathrm{L}}\left(\left\{\varphi_{S}^{t}\right\}_{t \geq 0}, S u_{e q}\right)=\operatorname{dim}_{\mathrm{L}}\left(\varphi_{S}^{t}, S u_{e q}\right), \quad \forall t>0 .
$$

Also, if for a certain $t=t^{c r}>0$ the maximum of finite-time local Lyapunov dimensions $\operatorname{dim}_{\mathrm{L}}\left(\varphi_{S}^{t^{c r}}, w\right)$ is achieved 6 at the point $w_{e q}^{c r}=S u_{e q} \in S K$ :

$$
\operatorname{dim}_{\mathrm{L}}\left(\varphi_{S}^{t^{c r}}, w_{e q}^{c r}\right)=\sup _{w \in S K} \operatorname{dim}_{\mathrm{L}}\left(\varphi_{S}^{t^{c r}}, w\right)
$$

\footnotetext{
${ }^{6}$ In general, since the function $u \mapsto \operatorname{dim}\left(\varphi^{t}, u\right)$ is upper semi-continuous (see, e.g. 27, p.554]), there exists a critical point $u_{\mathrm{L}}(t) \in K$ (it may be not unique) such that $\sup _{u \in K} \operatorname{dim}_{\mathrm{L}}\left(\varphi^{t}, u\right)=\operatorname{dim}_{\mathrm{L}}\left(\varphi^{t}, u_{\mathrm{L}}(t)\right)$. An interesting question is whether there exists a critical path $\gamma^{c r}=\left\{\varphi^{t}\left(u_{\mathrm{L}}\left(t_{0}\right)\right), t \geq 0\right\}$ such that for each $t \geq 0$ one of the corresponding critical points belongs to the critical path: $\varphi^{t}\left(u_{\mathrm{L}}\left(t_{0}\right)\right)=u_{\mathrm{L}}(t)$, and, if so, whether the critical path is an equilibrium or a periodic solution. The last part of the question was formulated in [21, p.98, Question 1]. Taking into account (15) we can consider increasing sequence $t_{k} \rightarrow+\infty$ such that $\operatorname{dim}_{\mathrm{L}}\left(\varphi^{t_{k}}, u_{\mathrm{L}}\left(t_{k}\right)\right)$ is monotonically decreasing to $\inf _{t>0} \operatorname{dim}_{\mathrm{L}}\left(\varphi^{t}, u_{\mathrm{L}}(t)\right)=\operatorname{dim}_{\mathrm{L}}\left(\left\{\varphi^{t}\right\}_{t>0}, K\right)$. Since $K$ is a compact set, we can obtain a subsequence $t_{m}=t_{k_{m}} \rightarrow+\infty$ such that there exists a limit critical point $u_{\mathrm{L}}^{c r}: u_{\mathrm{L}}\left(t_{m}\right) \rightarrow u_{\mathrm{L}}^{c r} \in K$ as $m \rightarrow+\infty$. Thus we have $\operatorname{dim}_{\mathrm{L}}\left(\varphi^{t_{m}}, u_{\mathrm{L}}\left(t_{m}\right)\right) \searrow \operatorname{dim}_{\mathrm{L}}\left(\left\{\varphi^{t}\right\}_{t \geq 0}, K\right)$ and $u_{\mathrm{L}}\left(t_{m}\right) \rightarrow u_{\mathrm{L}}^{c r} \in K$ as $m \rightarrow+\infty$.
} 
then

$$
\operatorname{dim}_{\mathrm{H}} K \leq \operatorname{dim}_{\mathrm{L}}\left(\left\{\varphi^{t}\right\}_{t \geq 0}, K\right)=\operatorname{dim}_{\mathrm{L}}\left(\left\{\varphi_{S}^{t}\right\}_{t \geq 0}, S K\right)=\operatorname{dim}_{\mathrm{L}}\left(\varphi_{S}^{t^{c r}}, K\right)=\operatorname{dim}_{\mathrm{L}}\left(\varphi_{S}^{t^{c r}}, w_{e q}^{c r}\right) .
$$

Here $\operatorname{dim}_{\mathrm{L}}\left(\varphi_{S}^{t^{c r}}, w_{e q}^{c r}\right)=\operatorname{dim}_{\mathrm{L}}\left(\varphi_{S}, w_{e q}^{c r}\right)$ and it can be computed by (23) with $\operatorname{LE}_{i}\left(S J\left(u_{e q}\right) S^{-1}\right)=$ $\lambda_{i}\left(u_{e q}\right)$ for continuous-time dynamical systems: $\operatorname{dim}_{\mathrm{L}}\left(\varphi_{S}^{t^{c r}}, w_{e q}^{c r}\right)=\operatorname{dim}_{\mathrm{L}}^{\mathrm{KY}}\left(\left\{\lambda_{i}\left(u_{e q}\right)\right\}_{1}^{n}\right)$, and with $\mathrm{LE}_{i}\left(S J\left(u_{e q}\right) S^{-1}\right)=\ln \left|\lambda_{i}\left(u_{e q}\right)\right|$ for discrete-time dynamical systems: $\operatorname{dim}_{\mathrm{L}}\left(\varphi_{S}^{t^{c r}}, w_{e q}^{c r}\right)=\operatorname{dim}_{\mathrm{L}}^{\mathrm{KY}}\left(\left\{\ln \left|\lambda_{i}\left(u_{e q}\right)\right|\right\}_{1}^{n}\right)$.

This statement may be useful in the numerical calculation of the Lyapunov dimension for global attractors and B-attractors (which contain unstable equilibria) [13, 55].

Consider now the Leonov method of analytical estimation of the Lyapunov dimension and its connection with the invariance of the Lyapunov dimension with respect to diffeomorphisms. Following [46, 58, 60], we consider a special class of diffeomorphisms such that $D h(u)=p(u) S$, where $p: U \subseteq \mathbb{R}^{n} \rightarrow \mathbb{R}^{1}$ is a continuous scalar function and $S$ is a nonsingular $n \times n$ matrix. Below it will be shown that the multiplier of the type $p\left(\varphi^{t}(u)\right)(p(u))^{-1}$ in (32) plays the role of the Lyapunov-like functions. This multiplier can also be interpreted as the changes of Riemannian metrics [67].

Consider continuous-time dynamical systems. Let $\lambda_{i}\left(u_{0}, S\right)=\lambda_{i}\left(S \varphi^{t}\left(u_{0}\right)\right), i=1,2, \ldots, n$, be the eigenvalues of the symmetrized Jacobian matrix

$$
\frac{1}{2}\left(S J\left(u\left(t, u_{0}\right)\right) S^{-1}+\left(S J\left(u\left(t, u_{0}\right)\right) S^{-1}\right)^{*}\right)=\frac{1}{2}\left(J_{S}\left(w\left(t, w_{0}\right)\right)+J_{S}\left(w\left(t, w_{0}\right)\right)^{*}\right),
$$

ordered so that $\lambda_{1}\left(u_{0}, S\right) \geq \cdots \geq \lambda_{n}\left(u_{0}, S\right)$ for any $u_{0} \in U$. The following theorem is reformulation of results from [46, 61, 62].

Theorem 1. If there exist an integer $j \in\{1, \ldots, n-1\}$, a real $s \in[0,1]$, a differentiable scalar function $V: U \subseteq \mathbb{R}^{n} \rightarrow \mathbb{R}^{1}$, and a nonsingular $n \times n$ matrix $S$ such that

$$
\sup _{u \in K}\left(\lambda_{1}(u, S)+\cdots+\lambda_{j}(u, S)+s \lambda_{j+1}(u, S)+\dot{V}(u)\right)<0,
$$

where $\dot{V}(u)=(\operatorname{grad}(V))^{*} f(u)$, then

$$
\operatorname{dim}_{\mathrm{H}} K \leq \operatorname{dim}_{\mathrm{L}}\left(\left\{\varphi^{t}\right\}_{t \geq 0}, K\right) \leq \operatorname{dim}_{\mathrm{L}}\left(\varphi^{T}, K\right)<j+s
$$

for all sufficiently large $T>0$.

Proof. Let $p(u)=e^{V(u)(j+s)^{-1}}$. From the following relation [78] (see, also [10] [p.48])

$$
\omega_{j+s}\left(S D \varphi^{t}(u) S^{-1}\right) \leq \exp \left(\int_{0}^{t} \lambda_{1}\left(S \varphi^{\tau}(u)\right)+\cdots+\lambda_{j}\left(S \varphi^{\tau}(u)\right)+s \lambda_{j+1}\left(S \varphi^{\tau}(u)\right) d \tau\right)
$$

and the relation

$$
\left(p\left(\varphi^{t}(u)\right) p(u)^{-1}\right)^{j+s}=\exp \left(V\left(\varphi^{t}(u)\right)-V(u)\right)=\exp \left(\int_{0}^{t} \dot{V}\left(\varphi^{\tau}(u)\right) d \tau\right)
$$

we get

$$
\begin{aligned}
& \left(p\left(\varphi^{t}(u)\right) p(u)^{-1}\right)^{j+s} \omega_{j+s}\left(S D \varphi^{t}(u) S^{-1}\right) \leq \\
& \quad \leq \exp \left(\int_{0}^{t}\left(\lambda_{1}\left(S \varphi^{\tau}(u)\right)+\cdots+\lambda_{j}\left(S \varphi^{\tau}(u)\right)+s \lambda_{j+1}\left(S \varphi^{\tau}(u)\right)+\dot{V}\left(\varphi^{\tau}(u)\right)\right) d \tau\right) .
\end{aligned}
$$


Since $\varphi^{t}(u) \in K$ for any $u \in K$, by (37) for $t>0$ we have

$$
\max _{u \in K}\left(\left(p\left(\varphi^{t}(u)\right) p(u)^{-1}\right)^{j+s} \omega_{j+s}\left(S D \varphi^{t}(u) S^{-1}\right)\right)<1, \quad t>0 .
$$

Therefore by Corollary 2 with $H(u)=p(u) S$, where $p(u)=\left(e^{V(u)}\right)^{\frac{1}{j+s}}$, we get the assertion of the theorem.

Remark. The idea of the estimation of the Hausdorff dimension by the eigenvalues of symmetrized Jacobian matrix was developed in [20, 78] (e.g. for $\dot{V}(u) \equiv 0$, condition (37) is considered in [78]). The function $p(u)=e^{V(u)(j+s)^{-1}}$ was introduced in [60] $(\dot{V}(u)$ allows one to effectively estimate the partial sum of the eigenvalues) and matrix $S$ was introduced in [58, eq.(8)] (for the simplification of eigenvalues computation). Condition (37) is valid if $\dot{V}(u)$ is continuous and $\lambda_{1}(u, S)+\cdots+\lambda_{j}(u, S)+s \lambda_{j+1}(u, S)+\dot{V}(u)<0$ for all $u \in K$ (here we take into account that $J(u, S)$ and, thus, $\lambda_{i}(u, S)$ are continuous). The constancy of the signs of $V(u)$ or $\dot{V}(u)$ is not required in the theorem.

Next, we consider discrete-time dynamical systems. Let $\lambda_{i}\left(u_{0}, S\right)=\lambda_{i}\left(S \varphi^{t}\left(u_{0}\right)\right), i=1,2, \ldots, n$ be the positive square roots of the eigenvalues of the symmetrized Jacobian matrix (i.e. the singular values of the Jacobian matrix)

$$
\left(\left(S J\left(u\left(t, u_{0}\right)\right) S^{-1}\right)^{*} S J\left(u\left(t, u_{0}\right)\right) S^{-1}\right)=\left(J_{S}\left(w\left(t, w_{0}\right)\right)^{*} J_{S}\left(w\left(t, w_{0}\right)\right)\right),
$$

ordered so that $\lambda_{1}\left(u_{0}, S\right) \geq \cdots \geq \lambda_{n}\left(u_{0}, S\right)>0$ for any $u_{0} \in U$.

Theorem 2. If there exist an integer $j \in\{1, \ldots, n-1\}$, a real $s \in[0,1]$, a continuous scalar function $V: K \subseteq \mathbb{R}^{n} \rightarrow \mathbb{R}^{1}$, and a nonsingular $n \times n$ matrix $S$ such that

$$
\sup _{u \in K}\left(\ln \lambda_{1}(u, S)+\cdots+\ln \lambda_{j}(u, S)+s \ln \lambda_{j+1}(u, S)+(V(\varphi(u))-V(u))\right)<0,
$$

then

$$
\operatorname{dim}_{\mathrm{H}} K \leq \operatorname{dim}_{\mathrm{L}}\left(\left\{\varphi^{t}\right\}_{t \geq 0}, K\right) \leq \operatorname{dim}_{\mathrm{L}}\left(\varphi^{T}, K\right)<j+s
$$

for all sufficiently large $T>0$.

Proof. By (9) for $D \varphi_{S}^{t}(w)=S D \varphi^{t}(u) S^{-1}=\prod_{\tau=0}^{t-1}\left(S J\left(u\left(\tau, u_{0}\right)\right) S^{-1}\right)$ we have

$$
\omega_{j+s}\left(S D \varphi^{t}(u) S^{-1}\right) \leq \prod_{\tau=0}^{t-1} \omega_{j+s}\left(S J\left(u\left(\tau, u_{0}\right)\right) S^{-1}\right)
$$

Therefore we get (the discrete analog of (38))

$$
\omega_{j+s}\left(S D \varphi^{t}(u) S^{-1}\right) \leq \prod_{\tau=0}^{t-1} \lambda_{1}\left(S \varphi^{\tau}(u)\right) \cdots \lambda_{j}\left(S \varphi^{\tau}(u)\right)\left(\lambda_{j+1}\left(S \varphi^{\tau}(u)\right)\right)^{s} .
$$

Let $p(u)=e^{V(u)(j+s)^{-1}}$. By the relation

$$
\left(p\left(\varphi^{t}(u)\right) p(u)^{-1}\right)^{j+s}=\exp \left(V\left(\varphi^{t}(u)\right)-V(u)\right)=\exp \left(\sum_{\tau=0}^{t-1} V\left(\varphi^{\tau+1}(u)\right)-V\left(\varphi^{\tau}(u)\right)\right)
$$


we obtain

$$
\begin{aligned}
& \ln \left(p\left(\varphi^{t}(u)\right) p(u)^{-1}\right)^{j+s}+\ln \omega_{j+s}\left(S D \varphi^{t}(u) S^{-1}\right) \leq \\
& \quad \leq \sum_{\tau=0}^{t-1}\left(\ln \lambda_{1}\left(S \varphi^{\tau}(u)\right)+\cdots+\ln \lambda_{j}\left(S \varphi^{\tau}(u)\right)+s \ln \lambda_{j+1}\left(S \varphi^{\tau}(u)\right)+V\left(\varphi\left(\varphi^{\tau}(u)\right)\right)-V\left(\varphi^{\tau}(u)\right)\right) .
\end{aligned}
$$

Since $\varphi^{t}(u) \in K$ for any $u \in K$, by (41) and Corollary 2 with $H(u)=p(u) S$, where $p(u)=$ $\left(e^{V(u)}\right)^{\frac{1}{j+s}}$, we get the assertion of the theorem.

Corollary 3. If conditions of Theorems 1 or 2 are valid for all $\bar{d}=(j+s) \in(d, n]$, and at an equilibrium point $u_{e q}^{c r} \equiv \varphi^{t}\left(u_{e q}^{c r}\right)$ the relation

$$
\operatorname{dim}_{\mathrm{L}}\left(\left\{\varphi^{t}\right\}_{t \geq 0}, u_{e q}^{c r}\right)=d
$$

holds, then for any invariant set $K \supset u_{e q}^{c r}$ from (35) we get the formula of exact Lyapunov dimension

$$
\operatorname{dim}_{\mathrm{H}} K \leq \operatorname{dim}_{\mathrm{L}}\left(\left\{\varphi^{t}\right\}_{t \geq 0}, K\right)=\operatorname{dim}_{\mathrm{L}}\left(\left\{\varphi^{t}\right\}_{t \geq 0}, u_{e q}^{c r}\right)=d
$$

In [9, 72] it is demonstrated how a technique similar to the above can be effectively used to derive constructive upper bounds of the topological entropy of dynamical systems.

Example. Consider the Henon map $\varphi_{\text {Henon }}: \mathbb{R}^{2} \rightarrow \mathbb{R}^{2}$

$$
\left(\begin{array}{l}
x \\
y
\end{array}\right) \mapsto\left(\begin{array}{l}
a+b y-x^{2} \\
x
\end{array}\right)
$$

where $a>0, b \in(0,1)$ are the parameters of mapping. The stationary points $\left(x_{ \pm}, x_{ \pm}\right)$of this map are the following: $x_{ \pm}=\frac{1}{2}\left(b-1 \pm \sqrt{(b-1)^{2}+4 a}\right)$. Following [46] we consider

$$
S=\left(\begin{array}{cc}
1 & 0 \\
0 & \sqrt{b}
\end{array}\right), \gamma=\frac{1}{\left(b-1-2 x_{-}\right) \sqrt{x_{-}^{2}+b}}, s \in[0,1) .
$$

In this case

$$
S J((x, y)) S^{-1}=\left(\begin{array}{cc}
-2 x & \sqrt{b} \\
\sqrt{b} & 0
\end{array}\right), \lambda_{1}((x, y), S)=\left(\sqrt{x^{2}+b}+|x|\right), \lambda_{2}((x, y), S)=\frac{b}{\lambda_{1}((x, y), S)} .
$$

If we take $V((x, y))=\gamma(1-s)(x+b y)$, then condition (41) with $j=1$ and

$$
s>s^{*}=\frac{\ln \left|\lambda_{1}\left(\left(x_{-}, x_{-}\right), S\right)\right|}{|\ln b-\ln | \lambda_{1}\left(\left(x_{-}, x_{-}\right), S\right)||}
$$

is satisfied for all $(x, y) \in \mathbb{R}^{2}$ and we need not localize the set $K$ in the phase space. By Proposition 3 and (23), at the equilibrium point $u_{e q}^{c r}=\left(x_{-}, x_{-}\right)$we have

$$
\operatorname{dim}_{\mathrm{L}}\left(\left\{\varphi_{\text {Henon }}^{t}\right\}_{t \geq 0},\left(x_{-}, x_{-}\right)\right)=\operatorname{dim}_{\mathrm{L}}^{\mathrm{KY}}\left(\left\{\ln \lambda_{i}\left(x_{-}, x_{-}\right)\right\}_{1}^{2}\right)=1+s^{*} .
$$

Therefore, for a bounded invariant set $K_{\text {Henon }}^{B} \ni\left(x_{-}, x_{-}\right)$(e.g. B-attractor) we have [46]

$$
\operatorname{dim}_{\mathrm{L}}\left(\left\{\varphi_{\text {Henon }}^{t}\right\}_{t \geq 0}, K_{\text {Henon }}^{B}\right)=\operatorname{dim}_{\mathrm{L}}\left(\left\{\varphi_{\text {Henon }}^{t}\right\}_{t \geq 0},\left(x_{-}, x_{-}\right)\right)=1+\frac{\ln \left|\lambda_{1}\left(\left(x_{-}, x_{-}\right), S\right)\right|}{|\ln b-\ln | \lambda_{1}\left(\left(x_{-}, x_{-}\right), S\right)||}
$$


Here for $a=1.4$ and $b=0.3$ we have $\operatorname{dim}_{\mathrm{L}}\left(\left\{\varphi_{\text {Henon }}^{t}\right\}_{t \geq 0}, K_{\text {Henon }}^{B}\right)=1.495 \ldots$. Remark that numerical localization of attractor 7 by the square: $-1.8 \leq x, y \leq 1.8$, allows one to estimate directly the singular values of $J(x, y)$ and obtain the estimation $\operatorname{dim}_{\mathrm{L}}\left(\left\{\varphi_{\text {Henon }}^{t}\right\}_{t \geq 0}, K_{\text {Henon }}^{B}\right) \leq 1.523$ [32] (this approach corresponds to the use of $V(u) \equiv 1$ in (41)). For attractor $K_{\text {Henon }}$ (which does not include equilibria) it is known from numerical experiments [74] that $\operatorname{dim}_{\mathrm{H}} K_{\text {Henon }} \approx$ 1.261 is in good agreement 8 with $\operatorname{dim}_{\mathrm{L}}\left(\left\{\varphi_{\text {Henon }}^{t}\right\}_{t \geq 0}, K_{\text {Henon }}\right) \approx 1.264$; algorithm from [55] gives $\operatorname{dim}_{\mathrm{L}}\left(\left\{\varphi_{\text {Henon }}^{t}\right\}_{t \geq 0}, K_{\text {Henon }}\right) \approx 1.262$.

\section{Conclusion}

In this work the Leonov method, based on the direct Lyapunov method with special Lyapunovlike functions, is derived from the invariance of the Lyapunov dimension with respect to diffeomorphisms. The advantage of this method is that it allows one to estimate the Lyapunov dimension of invariant sets without localization of the set in the phase space and, in some cases, to get effectively exact Lyapunov dimension formulas [46, 47, 50 53, 59, 64] (another approaches for rigorous derivation of exact Lyapunov dimension formulas are demonstrated, e.g. in [19, 23, 75]).

Remark that while the notion of local Lyapunov dimension is natural for the maps, a rigorous introduction of this notion for the dynamical systems is a challenging task. The above consideration is based on the Douady-Oesterlé theorem on the Lyapunov dimension of maps only. The results on the Lyapunov dimension of dynamical systems, developed by Constantin, Eden, Foiaş, and Temam in [14, 23, 24], have not been applied. In the definition of the Lyapunov dimension of the dynamical system $\left\{\varphi^{t}\right\}_{t \geq 0}$ (see (11) and (18)) they consider $\left(\omega_{d}\left(D \varphi^{t}(u)\right)\right)^{1 / t}$ instead of $\omega_{d}\left(D \varphi^{t}(u)\right)$ and apply the theory of positive operators [12] (see also [29]) to prove the existence of a critical point $9 u_{\mathrm{E}}^{c r}$ (which may be not unique), where the corresponding global Lyapunov dimension achieves maximum (see [23]):

$$
\begin{aligned}
\operatorname{dim}_{\mathrm{L}}^{\mathrm{E}}\left(\left\{\varphi^{t}\right\}_{t \geq 0}, K\right) & =\inf \left\{d \in[0, n]: \lim _{t \rightarrow+\infty} \max _{u \in K} \ln \left(\omega_{d}\left(D \varphi^{t}(u)\right)\right)^{1 / t}<0\right\}= \\
& =\inf \left\{d \in[0, n]: \limsup _{t \rightarrow+\infty} \ln \left(\omega_{d}\left(D \varphi^{t}\left(u_{\mathrm{E}}^{c r}\right)\right)\right)^{1 / t}<0\right\}=\operatorname{dim}_{\mathrm{L}}^{\mathrm{E}}\left(\left\{\varphi^{t}\right\}_{t \geq 0}, u_{\mathrm{E}}^{c r}\right),
\end{aligned}
$$

and, thus, rigorously justify the use of the local Lyapunov dimension $\operatorname{dim}_{\mathrm{L}}^{\mathrm{E}}\left(\left\{\varphi^{t}\right\}_{t \geq 0}, u\right)$.

\section{Acknowledgments}

The author would like to thank Alp Eden, Gennady Leonov, Joseph Oesterlé, Volker Reitmann, and James Yorke for their comments. The work was supported by Russian Science Foundation

7 The classical Henon attractor is not global, but it is self-excited with respect to both equilibria and, thus, can be visualized numerically by a trajectory with the initial data in their vicinities (i.e. the basin of attraction intersects with small neighborhoods of the equilibria; if there are no such equilibria, the attractor is called a hidden attractor [41, 49, 55, 57]). Hidden attractors can be found in the Henon map with negative $b$ (see, e.g. $a=1.63$, $b=-0.138)$. A conjecture on the Lyapunov dimension of self-excited attractors is that for "typical" systems the Lyapunov dimension of self-excited attractor is less then the Lyapunov dimension of one of the unstable equilibria, the unstable manifold of which intersects with the basin of attraction and allows to visualize the attractor.

8 Examples are known where such a good agreement between these dimensions does not take place [76].

9 See also the corresponding Eden conjecture [21, p.98, Question 1]. From Fekete's lemma for the subadditive functions [38, pp.463-464] it follows that for any $d \in[0, n]$ there exists $\lim _{t \rightarrow+\infty} \sup _{u \in K}\left(\omega_{d}\left(D \varphi^{t}(u)\right)\right)^{1 / t}$. Remark, that for the definition (11) with $\omega_{d}\left(D \varphi^{t}(u)\right)$ the change of order of inf $t>0$ and $\sup _{u \in K}$ in (18), e.g. justification of the use of $\sup \lim \sup \operatorname{dim}_{L}\left(\varphi^{t}, u\right)$ instead of $\liminf _{t \rightarrow+\infty} \sup \operatorname{dim}_{L}\left(\varphi^{t}, u\right)$, is an open problem. One might check the coincidence $u \in K \quad t \rightarrow+\infty \quad t \rightarrow+\infty u \in K$ of $u_{\mathrm{L}}^{c r}$ and $u_{\mathrm{E}}^{c r}$. 
(project 14-21-00041) and Saint-Petersburg State University.

\section{References}

[1] Abarbanel, H., Brown, R., and Kennel, M. (1991). Variation of Lyapunov exponents on a strange attractor. Journal of Nonlinear Science, 1(2):175-199.

[2] Abarbanel, H., Brown, R., Sidorowich, J., and Tsimring, L. (1993). The analysis of observed chaotic data in physical systems. Reviews of Modern Physics, 65(4):1331-1392.

[3] Adler, R., Konheim, A., and McAndrew, M. (1965). Topological entropy. Transactions of the American Mathematical Society, 114(2):309?319.

[4] Aleksandrov, P. and Pasynkov, B. (1973). Introduction to Dimension Theory (in Russian). Nauka, Moscow.

[5] Augustova, P., Beran, Z., and Celikovsky, S. (2015). ISCS 2014: Interdisciplinary Symposium on Complex Systems, Emergence, Complexity and Computation (Eds.: A. Sanayei et al.), chapter On Some False Chaos Indicators When Analyzing Sampled Data, pages 249-258. Springer.

[6] Barreira, L. and Gelfert, K. (2011). Dimension estimates in smooth dynamics: a survey of recent results. Ergodic Theory and Dynamical Systems, 31:641-671.

[7] Barreira, L. and Schmeling, J. (2000). Sets of "Non-typical" points have full topological entropy and full Hausdorff dimension. Israel Journal of Mathematics, 116(1):29-70.

[8] Bogoliubov, N. and Krylov, N. (1937). La theorie generalie de la mesure dans son application a l'etude de systemes dynamiques de la mecanique non-lineaire. Ann. Math. II (in French) (Annals of Mathematics), 38(1):65-113.

[9] Boichenko, V. and Leonov, G. (1998). Lyapunov's direct method in estimates of topological entropy. Journal of Mathematical Sciences, 91(6):3370-3379.

[10] Boichenko, V. A., Leonov, G. A., and Reitmann, V. (2005). Dimension Theory for Ordinary Differential Equations. Teubner, Stuttgart.

[11] Bylov, B. E., Vinograd, R. E., Grobman, D. M., and Nemytskii, V. V. (1966). Theory of characteristic exponents and its applications to problems of stability (in Russian). Nauka, Moscow.

[12] Choquet, G. and Foias, C. (1975). Solution d'un probleme sur les iteres d'un operateur positif sur $C(K)$ et proprietes de moyennes associees. Annales de l'institut Fourier (in French), 25(3-4):109-129.

[13] Chueshov, I. (2015). Dynamics of Quasi-Stable Dissipative Systems. Springer.

[14] Constantin, P., Foias, C., and Temam, R. (1985). Attractors representing turbulent flows. Memoirs of the American Mathematical Society, 53(314).

[15] Cvitanović, P., Artuso, R., Mainieri, R., Tanner, G., and Vattay, G. (2012). Chaos: Classical and Quantum. Niels Bohr Institute, Copenhagen. http://ChaosBook.org.

[16] Czornik, A., Nawrat, A., and Niezabitowski, M. (2013). Lyapunov exponents for discrete time-varying systems. Studies in Computational Intelligence, 440:29-44. 
[17] Dellnitz, M. and Junge, O. (2002). Set oriented numerical methods for dynamical systems. In Handbook of Dynamical Systems, volume 2, pages 221-264. Elsevier Science.

[18] Dinaburg, E. (1971). On the relations among various entropy characteristics of dynamical systems. Mathematics of the USSR-Izvestiya, 5(2):337-378.

[19] Doering, C., Gibbon, J., Holm, D., and Nicolaenko, B. (1987). Exact Lyapunov dimension of the universal attractor for the complex Ginzburg-Landau equation. Phys. Rev. Lett., 59:29112914.

[20] Douady, A. and Oesterle, J. (1980). Dimension de Hausdorff des attracteurs. C.R. Acad. Sci. Paris, Ser. A. (in French), 290(24):1135-1138.

[21] Eden, A. (1989a). An abstract theory of L-exponents with applications to dimension analysis (PhD thesis). Indiana University.

[22] Eden, A. (1989b). Local Lyapunov exponents and a local estimate of Hausdorff dimension. ESAIM: Mathematical Modelling and Numerical Analysis - Modelisation Mathematique et Analyse Numerique, 23(3):405-413.

[23] Eden, A. (1990). Local estimates for the Hausdorff dimension of an attractor. Journal of Mathematical Analysis and Applications, 150(1):100-119.

[24] Eden, A., Foias, C., and Temam, R. (1991). Local and global Lyapunov exponents. Journal of Dynamics and Differential Equations, 3(1):133-177. [Preprint No. 8804, The Institute for Applied Mathematics and Scientific Computing, Indiana University, 1988].

[25] Farmer, J., Ott, E., and Yorke, J. (1983). The dimension of chaotic attractors. Physica D: Nonlinear Phenomena, 7(1-3):153 - 180.

[26] Frederickson, P., Kaplan, J., Yorke, E., and Yorke, J. (1983). The Liapunov dimension of strange attractors. Journal of Differential Equations, 49(2):185-207.

[27] Gelfert, K. (2003). Maximum local Lyapunov dimension bounds the box dimension. Direct proof for invariant sets on Riemannian manifolds. Z. Anal. Anwend., 22:553-568.

[28] Grassberger, P. and Procaccia, I. (1983). Measuring the strangeness of strange attractors. Physica D: Nonlinear Phenomena, 9(1-2):189-208.

[29] Gundlach, V. and Steinkamp, O. (2000). Products of random rectangular matrices. Mathematische Nachrichten, 212(1):51-76.

[30] Hegger, R., Kantz, H., and Schreiber, T. (1999). Practical implementation of nonlinear time series methods: The TISEAN package. Chaos, 9:413-435.

[31] Horn, R. and Johnson, C. (1994). Topics in Matrix Analysis. Cambridge University Press, Cambridge.

[32] Hunt, B. (1996). Maximum local Lyapunov dimension bounds the box dimension of chaotic attractors. Nonlinearity, 9(4):845-852.

[33] Hurewicz, W. and Wallman, H. (1941). Dimension Theory. Princeton University Press, Princeton. 
[34] Izobov, N. A. (2012). Lyapunov exponents and stability. Cambridge Scientific Publischers, Cambridge.

[35] Kaplan, J., Mallet-Paret, J., and Yorke, J. (1984). The Lyapunov dimension of a nowhere differentiable attracting torus. Ergodic Theory and Dynamical Systems, 4:261-281.

[36] Kaplan, J. L. and Yorke, J. A. (1979). Chaotic behavior of multidimensional difference equations. In Functional Differential Equations and Approximations of Fixed Points, pages 204-227. Springer, Berlin.

[37] Kolmogorov, A. (1959). On entropy per unit time as a metric invariant of automorphisms. Dokl. Akad. Nauk SSSR (In Russian), 124(4):754-755.

[38] Kuczma, M. and Gilányi, A. (2009). An Introduction to the Theory of Functional Equations and Inequalities: Cauchy's Equation and Jensen's Inequality. Birkhäuser Basel.

[39] Kunze, M. and Kupper, T. (2001). Non-smooth dynamical systems: An overview. In Ergodic Theory, Analysis, and Efficient Simulation of Dynamical Systems, pages 431-452. Springer.

[40] Kuratowski, K. (1966). Topology. Academic press, New York.

[41] Kuznetsov, N. (2016). Hidden attractors in fundamental problems and engineering models. A short survey. Lecture Notes in Electrical Engineering, 371:13-25. (Plenary lecture at AETA 2015: Recent Advances in Electrical Engineering and Related Sciences).

[42] Kuznetsov, N., Alexeeva, T., and Leonov, G. (2016). Invariance of Lyapunov exponents and Lyapunov dimension for regular and irregular linearizations. Nonlinear Dynamics, 85(1):195201.

[43] Kuznetsov, N. and Leonov, G. (2005). On stability by the first approximation for discrete systems. In 2005 International Conference on Physics and Control, PhysCon 2005, volume Proceedings Volume 2005, pages 596-599. IEEE.

[44] Kuznetsov, N., Mokaev, T., and Vasilyev, P. (2014). Numerical justification of Leonov conjecture on Lyapunov dimension of Rossler attractor. Commun Nonlinear Sci Numer Simulat, 19:1027-1034.

[45] Ledrappier, F. (1981). Some relations between dimension and Lyapounov exponents. Communications in Mathematical Physics, 81(2):229-238.

[46] Leonov, G. (2002). Lyapunov dimension formulas for Henon and Lorenz attractors. St.Petersburg Mathematical Journal, 13(3):453-464.

[47] Leonov, G., Alexeeva, T., and Kuznetsov, N. (2015a). Analytic exact upper bound for the Lyapunov dimension of the Shimizu-Morioka system. Entropy, 17(7):5101-5116.

[48] Leonov, G. and Kuznetsov, N. (2007). Time-varying linearization and the Perron effects. International Journal of Bifurcation and Chaos, 17(4):1079-1107.

[49] Leonov, G. and Kuznetsov, N. (2013). Hidden attractors in dynamical systems. From hidden oscillations in Hilbert-Kolmogorov, Aizerman, and Kalman problems to hidden chaotic attractors in Chua circuits. International Journal of Bifurcation and Chaos, 23(1). art. no. 1330002. 
[50] Leonov, G. and Kuznetsov, N. (2015). On differences and similarities in the analysis of Lorenz, Chen, and Lu systems. Applied Mathematics and Computation, 256:334-343.

[51] Leonov, G., Kuznetsov, N., Korzhemanova, N., and Kusakin, D. (2015b). Estimation of Lyapunov dimension for the Chen and Lu systems. arXiv, http://arxiv.org/pdf/1504.04726v1.pdf.

[52] Leonov, G., Kuznetsov, N., Korzhemanova, N., and Kusakin, D. (2015c). The Lyapunov dimension formula for the global attractor of the Lorenz system. arXiv, http://arxiv.org/pdf/1508.07498v1.pdf.

[53] Leonov, G., Kuznetsov, N., Korzhemanova, N., and Kusakin, D. (2015d). Lyapunov dimension formula of attractors in the Tigan and Yang systems. arXiv:1510.01492v1, http://arxiv.org/pdf/1510.01492v1.pdf.

[54] Leonov, G., Kuznetsov, N., Korzhemanova, N., and Kusakin, D. (2016). Lyapunov dimension formula for the global attractor of the Lorenz system. Communications in Nonlinear Science and Numerical Simulation, 41:84-103.

[55] Leonov, G., Kuznetsov, N., and Mokaev, T. (2015e). Homoclinic orbits, and self-excited and hidden attractors in a Lorenz-like system describing convective fluid motion. Eur. Phys. J. Special Topics, 224(8):1421-1458.

[56] Leonov, G., Kuznetsov, N., and Mokaev, T. (2015f). The Lyapunov dimension formula of self-excited and hidden attractors in the Glukhovsky-Dolzhansky system. arXiv:1509.09161. http://arxiv.org/pdf/1509.09161v1.pdf.

[57] Leonov, G., Kuznetsov, N., and Vagaitsev, V. (2011a). Localization of hidden Chua's attractors. Physics Letters A, 375(23):2230-2233.

[58] Leonov, G. and Lyashko, S. (1993). Eden's hypothesis for a Lorenz system. Vestnik St. Petersburg University: Mathematics, 26(3):15-18. [Transl. from Russian: Vestnik SanktPeterburgskogo Universiteta. Ser 1. Matematika, 26(3), 14-16].

[59] Leonov, G. and Poltinnikova, M. (2005). On the Lyapunov dimension of the attractor of Chirikov dissipative mapping. AMS Translations. Proceedings of St.Petersburg Mathematical Society. Vol. X, 224:15-28.

[60] Leonov, G. A. (1991). On estimations of Hausdorff dimension of attractors. Vestnik St. Petersburg University: Mathematics, 24(3):38-41. [Transl. from Russian: Vestnik Leningradskogo Universiteta. Mathematika, 24(3), 1991, pp. 41-44].

[61] Leonov, G. A. (2008). Strange attractors and classical stability theory. St.Petersburg University Press, St.Petersburg.

[62] Leonov, G. A. (2012). Lyapunov functions in the attractors dimension theory. Journal of Applied Mathematics and Mechanics, 76(2):129-141.

[63] Leonov, G. A. and Boichenko, V. A. (1992). Lyapunov's direct method in the estimation of the Hausdorff dimension of attractors. Acta Applicandae Mathematicae, 26(1):1-60.

[64] Leonov, G. A., Pogromsky, A. Y., and Starkov, K. E. (2011b). Dimension formula for the Lorenz attractor. Physics Letters A, 375(8):1179-1182. 
[65] Lyapunov, A. M. (1892). The General Problem of the Stability of Motion (in Russian). Kharkov. [English transl.: Academic Press, NY, 1966].

[66] Millionschikov, V. M. (1976). A formula for the entropy of smooth dynamical systems. Differencial'nye Uravenija (in Russian), 12(12):2188-2192, 2300.

[67] Noack, A. and Reitmann, V. (1996). Hausdorff dimension estimates for invariant sets of time-dependent vector fields. Z. Anal. Anwend., 15:457-473.

[68] Oseledets, V. (1968). A multiplicative ergodic theorem. Characteristic Ljapunov, exponents of dynamical systems. Trudy Moskovskogo Matematicheskogo Obshchestva (in Russian), 19:179210.

[69] Ott, E., Withers, W., and Yorke, J. (1984). Is the dimension of chaotic attractors invariant under coordinate changes? Journal of Statistical Physics, 36(5-6):687-697.

[70] Ott, W. and Yorke, J. (2008). When Lyapunov exponents fail to exist. Phys. Rev. E, 78:056203.

[71] Pesin, Y. (1977). Characteristic Lyapunov exponents and smooth ergodic theory. Russian Mathematical Surveys, 32(4):55-114.

[72] Pogromsky, A. Y. and Matveev, A. S. (2011). Estimation of topological entropy via the direct Lyapunov method. Nonlinearity, 24(7):1937-1959.

[73] Rosenstein, M., Collins, J., and De Luca, C. (1993). A practical method for calculating largest Lyapunov exponents from small data sets. Physica D: Nonlinear Phenomena, 65(1-2):117-134.

[74] Russel, D., Hanson, J., and Ott, E. (1980). Dimension of strange attractors. Physical Review Letters, 45(14):1175-1178.

[75] Schmeling, J. (1998). A dimension formula for endomorphisms - the Belykh family. Ergodic Theory and Dynamical Systems, 18:1283-1309.

[76] Sell, G. (1989). The Connection between Infinite Dimensional and Finite Dimensional Dynamical Systems (Eds.: Nicolaenko, B. and Foias, C. and Temam, R.), chapter Hausdorff and Lyapunov dimensions for gradient systems, pages 85-92. American Mathematical Society.

[77] Sinai, Y. (1959). On the notion of entropy of dynamical systems. Dokl. Akad. Nauk SSSR (In Russian), 124(4):768-771.

[78] Smith, R. (1986). Some application of Hausdorff dimension inequalities for ordinary differential equation. Proc. Royal Society Edinburg, 104A:235-259.

[79] Tempkin, J. and Yorke, J. (2007). Spurious Lyapunov exponents computed from data. SIAM Journal on Applied Dynamical Systems, 6(2):457-474.

[80] Wolf, A., Swift, J. B., Swinney, H. L., and Vastano, J. A. (1985). Determining Lyapunov exponents from a time series. Physica D: Nonlinear Phenomena, 16(D):285-317.

[81] Young, L.-S. (2013). Mathematical theory of Lyapunov exponents. Journal of Physics A: Mathematical and Theoretical, 46(25):254001. 